\title{
Role of macrophage sialoadhesin in host defense against the sialylated pathogen group B Streptococcus
}

\author{
Yung-Chi Chang • Joshua Olson • Aaron Louie • \\ Paul R. Crocker • Ajit Varki • Victor Nizet
}

Received: 12 February 2014 /Revised: 23 March 2014 / Accepted: 14 April 2014 / Published online: 3 May 2014

(C) Springer-Verlag Berlin Heidelberg 2014

\begin{abstract}
Several bacterial pathogens decorate their surfaces with sialic acid (Sia) residues within cell wall components or capsular exopolysaccharides. Sialic acid expression can promote bacterial virulence by blocking complement activation or by engagement of inhibitory sialic acid-binding immunoglobulin-like lectins (Siglecs) on host leukocytes. Expressed at high levels on splenic and lymph node macrophages, sialoadhesin (Sn) is a unique
\end{abstract}

Electronic supplementary material The online version of this article (doi:10.1007/s00109-014-1157-y) contains supplementary material, which is available to authorized users.

Y.-C. Chang $\cdot$ A. Varki $(\bowtie) \cdot$ V. Nizet

Glycobiology Research and Training Center, University of

California, San Diego, La Jolla, CA 92093, USA

e-mail: a1varki@ucsd.edu

Y.-C. Chang $\cdot$ J. Olson $\cdot$ A. Louie $\cdot$ V. Nizet

Department of Pediatrics, University of California, San Diego, La Jolla, CA 92093, USA

A. Varki

Department of Cellular and Molecular Medicine, University of California, San Diego, La Jolla, CA 92093, USA

\section{A. Varki}

Department of Medicine, University of California, San Diego, La Jolla, CA 92093, USA

\section{Nizet}

Skaggs School of Pharmacy and Pharmaceutical Sciences,

University of California, San Diego, La Jolla, CA 92093, USA

\section{P. R. Crocker}

Division of Cell Signalling and Immunology, College of Life

Sciences, University of Dundee, Dundee, UK

\section{Nizet $(\bowtie)$}

Division of Pediatric Pharmacology and Drug Discovery, University of California, San Diego, 9500 Gilman Drive, Mail Code 0687, La Jolla, CA 92093-0687, USA

e-mail: vnizet@ucsd.edu
Siglec with an elongated structure that lacks intracellular signaling motifs. Sialoadhesin allows macrophage to engage certain sialylated pathogens and stimulate inflammatory responses, but the in vivo significance of sialoadhesin in infection has not been shown. We demonstrate that macrophages phagocytose the sialylated pathogen group B Streptococcus (GBS) and increase bactericidal activity via sialoadhesin-sialic-acid-mediated recognition. Sialoadhesin expression on marginal zone metallophillic macrophages in the spleen trapped circulating GBS and restricted the spread of the GBS to distant organs, reducing mortality. Specific IgM antibody responses to GBS challenge were also impaired in sialoadhesin-deficient mice. Thus, sialoadhesin represents a key bridge to orchestrate innate and adaptive immune defenses against invasive sialylated bacterial pathogens.

\section{Key message}

- Sialoadhesin is critical for macrophages to phagocytose and clear GBS.

- Increased GBS organ dissemination in the sialoadhesindeficient mice.

- Reduced anti-GBS IgM production in the sialoadhesindeficient mice.

Keywords Metallophillic macrophage $\cdot$ Antibody $\cdot$ Siglec . Sialic acid

\section{Introduction}

The spleen is the largest secondary lymphoid organ in our body, receiving $5 \%$ of the cardiac output in blood perfusion, and capable of screening the entire blood volume for pathogens within 20-30 min [1]. The crucial role of the spleen in blood filtering is evident in asplenic individuals, who are particularly vulnerable to sepsis with encapsulated bacteria such as Neisseria meningitidis, Streptococcus pneumoniae, 
and Haemophilus influenzae [2,3]. Opening of the arterial blood stream into the marginal sinuses of the spleen reduces blood flow so that pathogens in the systemic circulation can be efficiently phagocytosed by marginal zone macrophages (MZMs) and marginal metallophilic macrophages (MMMs) $[4,5]$. Depletion of MZMs and MMMs can result in pathogens escaping to the blood, potentially triggering uncontrolled bacteremia and sepsis $[6,7]$.

MMMs, but typically not MZMs, express high levels of sialoadhesin ( $\mathrm{Sn}$, sialic acid-binding immunoglobulin-like lectin-1 (Siglec-1), CD169) and form an inner ring bordering the marginal zone and the white pulp follicular areas. Among Siglecs, Sn is unique in possessing 17 immunoglobulin-like extracellular domains that may extend its length $\sim 40 \mathrm{~nm}$ beyond the cell surface and recognize sialylated ligands found on many sialylated pathogens, such as sialylated enveloped viruses [8-10], bacteria [11, 12], and protozoa [13]. More recently, Sn has been shown to capture killed sialylated Campylobacter jejuni and able to promote rapid proinflammatory cytokine and type I interferon responses [14]. However, the consequences of Sn-dependent recognition of an invasive sialylated bacteria pathogen on infection outcome have not been addressed.

In this work, we used the sialylated pathogen group B Streptococcus (GBS), a leading cause of human neonatal sepsis and meningitis, as a model for in vitro and in vivo analysis of Sn function. Specifically, we studied whether expression of $\mathrm{Sn}$ in MMMs could facilitate splenic trapping of GBS during early infection and/or influence later phase humoral responses to coordinately combat this invasive blood-borne pathogen.

\section{Materials and methods}

Bacteria and growth condition

GBS of serotypes Ia (A909), Ib (UAB), II (DK23), III (COH1, K79 and D136), and VI (NT-6) are human neonate isolates. $\mathrm{COH} 1 \Delta$ NeuA mutant and isogenic $\mathrm{COH} 1$ mutants expressing different levels of $O$-acetylation on the terminal sialic acid (Sia) were previously described $[15,16]$. GBS were grown in Todd-Hewitt broth (Difco, BD Diagnostics) at $37{ }^{\circ} \mathrm{C}$ without shaking. For functional assays, bacteria were cultivated at $37^{\circ} \mathrm{C}$ to early log phase and resuspended to $\mathrm{OD}_{600}$ of 0.4 in appropriate buffer to the desired concentrations.

Culture of mouse bone marrow-derived macrophages (MBDMs) and in vitro stimulation with GBS

MBDMs were derived from marrow cells collected from femur and tibia and cultured with conditioned media containing macrophage colony-stimulating factors (M-CSF) for
7 days. MBDMs were then seeded onto 24 -well plates at $5 \times$ $10^{5} /$ well with M-CSF (10 $\mathrm{ng} / \mathrm{ml}, \mathrm{R} \& \mathrm{D}$ Systems) and interferon- $\alpha$ (500 U/ml, PBL IFN Source) to optimize Sn expression. For phagocytosis assays, $2.5 \times 10^{6}$ CFU GBS DK23 was added to $5 \times 10^{5}$ macrophages $(\mathrm{MOI}=5)$. Following a 30-min incubation, cells were washed, added medium containing penicillin $(5 \mu \mathrm{g} / \mathrm{ml})$ and gentamicin $(100 \mu \mathrm{g} / \mathrm{ml})$ to kill extracellular bacteria, detached by trypsin, lysed with $0.025 \%$ Triton X-100, and plated to enumerate intracellular bacteria. For bacterial killing, $10^{5}$ GBS DK23 was added to MBDMs at MOI $=0.2$ for $1 \mathrm{~h}$, followed by addition of $50 \mu \mathrm{l}$ of $0.6 \%$ Triton X-100 to lyse cells. Recovered GBS were plated for CFU enumeration. For experiments using Sn-neutralizing mAb (clone SER-4, $10 \mu \mathrm{g} / \mathrm{ml}$ ), the mAb was added to cells $10 \mathrm{~min}$ prior to inoculation with GBS. This neutralizing $\mathrm{mAb}$ has been shown to block the sialic-acid-mediated interaction between $\mathrm{Sn}$ and erythrocytes as well as the interaction between $\mathrm{Sn}$ and sialylated meningococcal lipopolysaccharide [11, 17].

Flow cytometry analysis of Sn expression

MBDMs were stimulated with IFN- $\alpha$ (500 U/ml), LPS (1 $\mu \mathrm{g} / \mathrm{ml}$, InvivoGen), lipoteichoic acid $(1 \mu \mathrm{g} / \mathrm{ml}$, InvivoGen), GBS capsule $(1 \mu \mathrm{g} / \mathrm{ml}$, purified as described [15]), or heat-killed GBS $\left(10^{8} \mathrm{CFU}\right)$ for 2 days. Cells were detached by $5 \mathrm{mM}$ EDTA/PBS, stained with FITC-conjugated rat anti-mouse Sn (clone 3D6, Serotec), and analyzed by flow cytometer (FACSCalibur, BD Biosciences).

Siglec-Fc binding assay

Siglec-Fc and FITC-labeled GBS binding studies were modified from a described method [18]. GBS was labeled with $0.1 \% \mathrm{FITC} / \mathrm{PBS}$ for $30 \mathrm{~min}$ at $37{ }^{\circ} \mathrm{C}$ with rotation. Ninetysix-well plates were coated with $1 \mu \mathrm{g} /$ well protein $\mathrm{A}$ in $50 \mathrm{mM}$ carbonate buffer ( $\mathrm{pH} 9.5$ ) overnight at $4{ }^{\circ} \mathrm{C}$, washed, and blocked with $1 \% \mathrm{BSA} / \mathrm{PBS}$ for $1 \mathrm{~h}$ at room temperature. Human and murine Sn-Fc $(0.5 \mu \mathrm{g})$ (produced as described [11]) was immobilized to individual wells for $\geq 3 \mathrm{~h}$ at room temperature. FITC-labeled GBS $\left(10^{7}\right)$ was added to each well, and a 96-well plate was centrifuged and incubated for $30 \mathrm{~min}$ at $37^{\circ} \mathrm{C}$. Initial fluorescence intensity was verified, and the residual fluorescence intensity (excitation, $488 \mathrm{~nm}$; emission, $530 \mathrm{~nm}$ ) was measured using a CytoFluor ${ }^{\mathrm{II}}$ fluorescence plate reader after extensive wash to remove unbound bacteria.

Immunofluorescence microscopy-based phagocytosis analysis

GBS DK23 was labeled with PKH26 (Sigma) per manufacturer's instruction. Labeled GBS $\left(2.5 \times 10^{6} \mathrm{CFU}\right)$ were added to $5 \times 10^{5}$ macrophages and incubated for $30 \mathrm{~min}$. Macrophages 
were washed, fixed, permeabilized, blocked with BSA, and stained with AlexaFluor $488^{\circledR}$ phalloidin (Life Technologies) and DAPI for $30 \mathrm{~min}$ at room temperature. Images were acquired using the Olympus FV1000 confocal microscope and FV1000 Viewer software.

Intravenous GBS infection and visualization of GBS in the spleen

All studies were approved by the UCSD Committee on the Use and Care of Animals and performed using accepted veterinary standards. Generation of Sn-deficient mice has previously been described [19]. GBS DK23 was labeled with 5-(and-6)-carboxyfluorescein (Invitrogen) per manufacturer's instructions. Mice were intravenously infected with $2 \times$ $10^{8} \mathrm{CFU}$ of labeled GBS via tail vein. Kidney, lung, and spleen were collected for CFU enumeration or frozen in OCT solution $1 \mathrm{~h}$ after infection. Spleen cryostat sections $(5 \mu \mathrm{m})$ were fixed in acetone and blocked with $1 \% \mathrm{BSA} /$ PBST and AVIDIN/BIOTIN blocking kit (Vector Laboratories). The sections were stained with rat $\mathrm{mAb}$ antimouse Sn (clone SER-4) with Alexa Fluor 647-conjugated goat anti-rat IgG (Life Technologies) followed by second-step staining with biotinylated rat $\mathrm{mAb}$ anti-mouse $\mathrm{F} 4 / 80$ (clone CI:A3-1, Serotec) or B220 (clone RA3-6B2, BD Biosciences) with Alexa Fluor 555-conjugated streptavidin (Life Technologies). Images were from an Olympus FV1000 confocal microscope using FV1000 Viewer software.

\section{GBS intraperitoneal infection mouse model}

Wild-type (WT) and Sn-deficient mice (10-12 weeks) were infected intraperitoneally with $10^{8} \mathrm{CFU}$ of GBS DK23 and bacterial $\mathrm{CFU}$ in the blood monitored $4 \mathrm{~h}$ post-infection. To detect anti-GBS antibody responses, mice (10-12 weeks) were first injected with $0.5 \mathrm{mg}$ of anti-Ly-6G mAb (clone 1A8, BioXcell) intraperitoneally to deplete neutrophils $24 \mathrm{~h}$ prior to infection with $2 \times 10^{7} \mathrm{CFU}$ of GBS DK23. Mouse survival was monitored twice per day for 14 days at which time mice were euthanized and their blood was collected for antibody titer analysis.

\section{ELISA of anti-GBS antibody isotypes}

Fifty microliters of GBS $\left(\mathrm{OD}_{600}=1.0\right)$ in $0.1 \mathrm{M}$ sodium carbonate buffer ( $\mathrm{pH} 9.5$ ) was added into 96-well plates, spun at 2,000 rpm for $10 \mathrm{~min}$, and incubated overnight at $4{ }^{\circ} \mathrm{C}$. GBScoated plates were blocked with $3 \%$ BSA/PBST for $1 \mathrm{~h}$ at room temperature and then mouse sera was added in 3-fold serial dilutions and incubated for $2 \mathrm{~h}$ at room temperature. Antibody titers were determined with horseradish peroxidaseconjugated immunoglobulin isotype-specific antibodies (Southern Biotechnology) and TMB substrate solution. OD
$450 \mathrm{~nm}$ was measured with a SpectraMax M3 microplate reader.

\section{ELISA of total antibody isotypes}

Ninety-six-well plates were coated with goat anti-mouse $\operatorname{IgG}+\operatorname{Ig} \mathrm{A}+\operatorname{IgM}$ (Southern Biotechnology, $0.5 \mu \mathrm{g} / \mathrm{well}$ in $0.1 \mathrm{M}$ sodium carbonate buffer, $\mathrm{pH} 9.5$ ) at $4{ }^{\circ} \mathrm{C}$ overnight. Plates were blocked with $3 \% \mathrm{BSA} / \mathrm{PBST}$ for $1 \mathrm{~h}$, and sera collected from GBS-infected mice was added (1:5,000 dilution for $\operatorname{IgM}$ and $\operatorname{IgA} ; 1: 50,000$ dilution for $\operatorname{IgG}$ ) and incubated for $2 \mathrm{~h}$ at room temperature. Antibodies were detected by horseradish peroxidase-conjugated immunoglobulin isotype-specific antibodies and TMB substrate solution. OD $450 \mathrm{~nm}$ was measured with a SpectraMax M3 microplate reader.

\section{Results}

Sialoadhesin (Sn) binds to sialylated group B Streptococcus (GBS), and its expression is upregulated by bacterial components and inflammatory stimuli

GBS is a leading cause of neonatal pneumonia and sepsis, and its surface capsular polysaccharide is invariably capped with a terminal $\alpha 2-3$-linked sialic acid (Sia) known to impair phagocytosis and dampen neutrophil bactericidal activities via engaging inhibitory Siglecs and to block complement deposition [18, 20-23]. Sn, a unique Siglec possessing 17 immunoglobulin-like extracellular domains, has been reported to recognize sialylated ligands found on many sialylated pathogens. We sought to determine if Sn could recognize GBS in a Sia-dependent manner as a defense strategy to counteract suppressive signals transduced by inhibitory Siglecs. Seven GBS strains (A909, UAB, DK23, COH1, K79, D136, and NT-6) tested here all bound to human $\mathrm{Sn}(\mathrm{hSn})$ and murine $\mathrm{Sn}$ $(\mathrm{mSn})$, but the Sia-negative $\mathrm{COH} 1 \Delta \mathrm{NeuA}$ mutant did not (Fig. 1a).

Expression of Sn on human monocytes is upregulated by IFN- $\alpha$ and agonists for Toll-like receptors [24]. In addition, under infection or pathological conditions, expression of $\mathrm{Sn}$ on circulating human monocytes and tissue macrophages can be increased and correlated to disease progression [24-29]. We observed that IFN- $\alpha$ significantly increased Sn expression on mouse bone marrow-derived macrophages (MBDMs), as did lipopolysaccharide (LPS), lipoteichoic acid (LTA), GBS capsular polysaccharide preparation, and heat-killed GBS (HK GBS) (Fig. 1b). Upregulation of Sn expression during infection could theoretically influence macrophage phagocytic and bactericidal activity against GBS. 
A GBS Binding

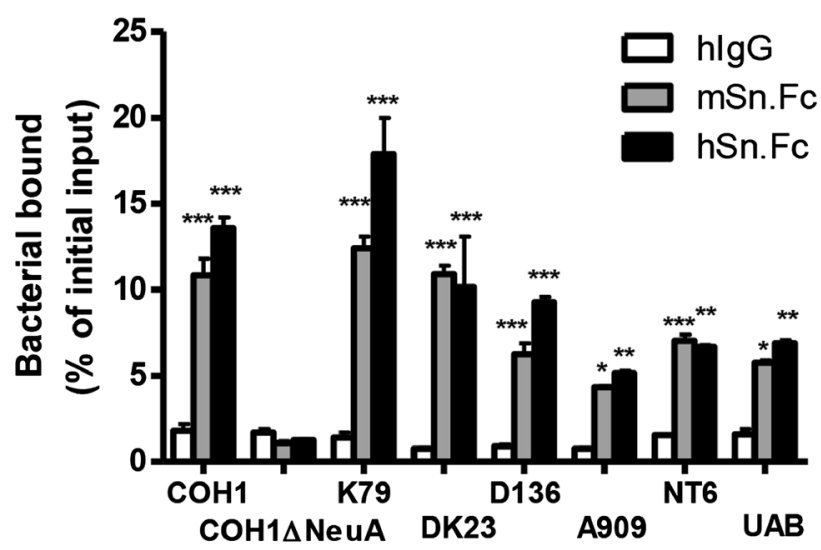

B
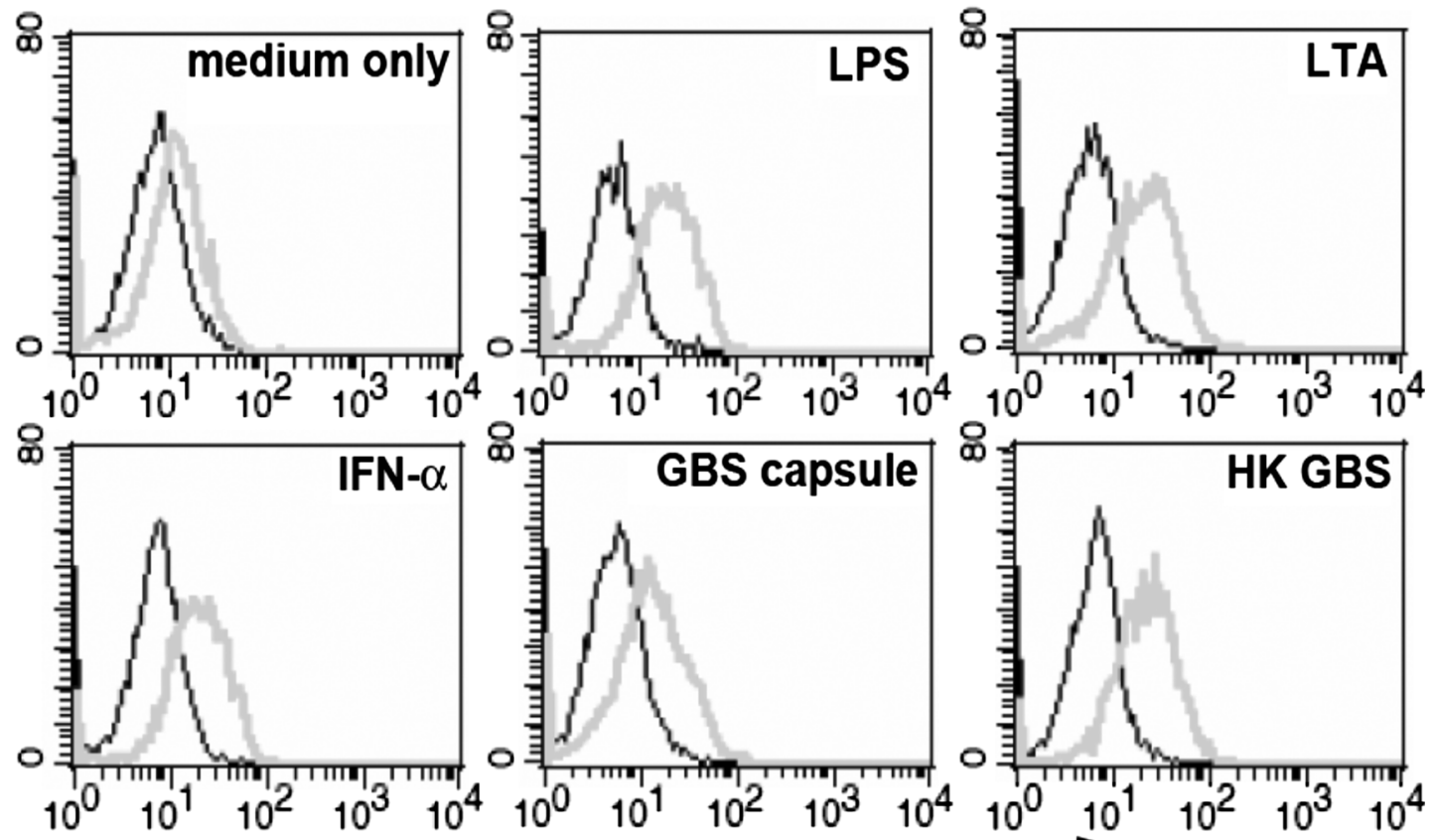

surface expression of sialoadhesin

Fig. 1 Sn binds group B Streptococcus (GBS) and is upregulated by inflammatory stimuli. a FITC-labeled GBS were added to plates coated with human Sn $(h S n)$, murine Sn $(m S n)$, or control human $\operatorname{IgG}(h \operatorname{Ig} G)$. Binding of bacteria to applied proteins was measured using a fluorescent plate reader after extensive washes. Data shown are means \pm SEM and the GBS binding difference between control and $\mathrm{Sn}$ was determined by twoway ANOVA with Bonferroni post-test. ${ }^{* * *} P<0.001 ; * * P<0.01$;
$* P<0.05$. Experiments have been done with three biological replicates and repeated twice. b MBDMs were stimulated with various bacterial components or IFN- $\alpha$ for 2 days, stained with anti-Sn mAb, and subjected to FACS analysis to examine Sn expression. Black thin line indicates isotype control; gray thick line indicates anti-Sn mAb. Representative data from one of the two reproducible independent experiments was shown

phagocytosis, adding a Sn-neutralizing mAb significantly reduced the ability of WT MBDMs to phagocytose GBS to the level of Sn-deficient cells (Fig. 2b). In addition, surviving GBS were also significantly increased in the infected Sn-deficient cells (Fig. 2c). To confirm if recognition of GBS capsular Sia by Sn specifically contributes to macrophage phagocytic and bactericidal activity, we studied another GBS serotype III strain, COH1, and isogenic mutants derived from this strain that either lack Sia 
A

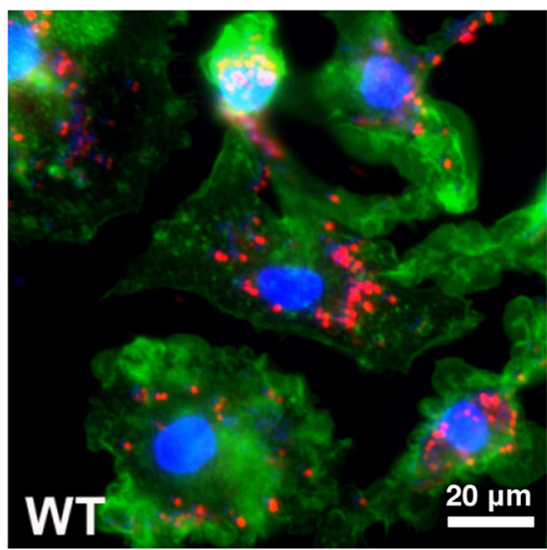

\section{B}

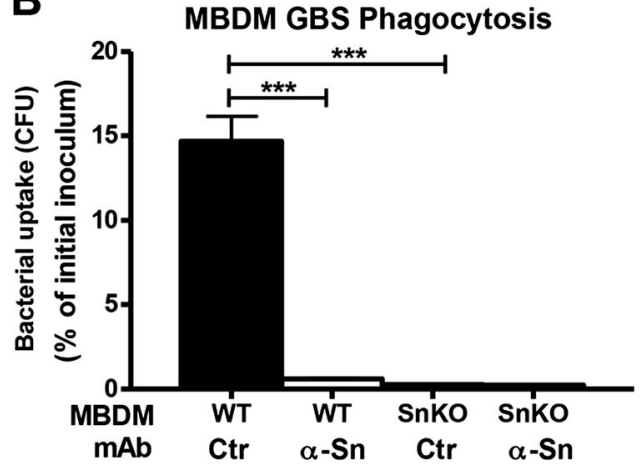

Fig. 2 Sn contributes to phagocytic and bactericidal activity against GBS. a Confocal microscopy images show significantly reduced phagocytosis of GBS in the Sn-deficient $(\mathrm{Sn} K O$ ) MBDMs. Macrophages were infected with GBS at MOI of 5 for 30 min and washed extensively before staining cells with actin. Red indicates GBS. Green indicates actin. Blue indicates nucleus. b Quantitative phagocytosis analysis. MBDMs were infected with GBS in the presence or absence of Sn-neutralizing mAb, followed by addition of antibiotics to only recover and enumerate

expression or express differing levels of Sia $O$-acetylation. $O$ acetylation is known to interfere with Siglec-mediated Sia binding $[15,16]$. WT MBDMs phagocytosed more WT GBS and mutant GBS with reduced Sia $O$-acetylation than did Sndeficient MBDMs. On the other hand, GBS lacking Sia on their capsule, or GBS with high level of $O$-acetylation on the capsule Sia, were phagocytosed equally by WT and Sn-deficient MBDMs (Supplementary Fig. 1A, B). Non-sialylated GBS and GBS with reduced capsular Sia $O$-acetylation levels were killed more efficiently by WT BMDMs than Sn-deficient macrophages (Supplementary Fig. 1C). Our findings demonstrate that $\mathrm{Sn}$ is critical for macrophage recognition of GBS and contributes to bactericidal activity against the sialylated pathogen.

Sn contributes to GBS capture and control of bacterial dissemination in vivo

Splenic MZ macrophages are known to actively sample and trap blood-borne pathogens. Since MMMs express high levels of Sn, we examined whether Sn facilitated MMM recognition

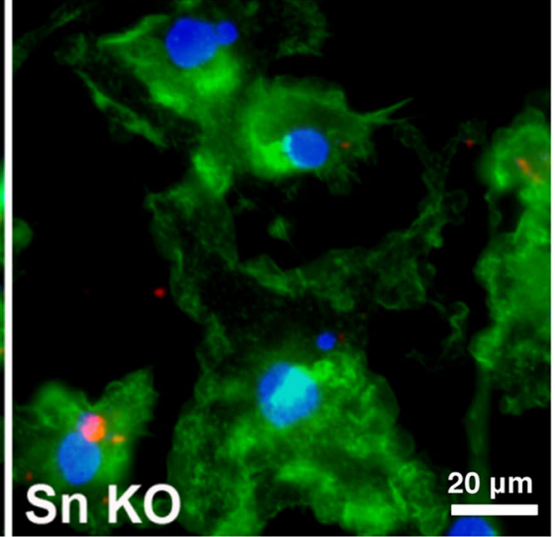

C

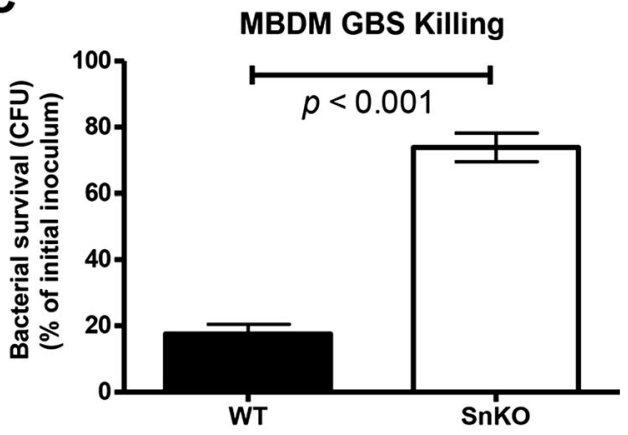

intracellular bacteria CFU. c Survival of GBS upon co-incubation with MBDMs. Macrophages were infected with GBS at MOI of 0.2, and surviving GBS was enumerated $1 \mathrm{~h}$ post-infection by serial plating. Difference between two groups was calculated one-way ANOVA with Tukey's multiple comparison test (b) or by unpaired $t$ test (c). $* * * P<0.001$. Representative image (a) and data (b and c) were shown from three independent experiments

of GBS in vivo. 5-(and-6)-carboxyfluorescein-labeled GBS were injected intravenously into mice, and the distribution of GBS was visualized in the spleen co-stained with $\mathrm{mAb}$ antimouse F4/80 (red pulp macrophage marker) and B220 (B cell marker). Strong co-localization of GBS and $\mathrm{Sn}^{+}$MMMs was observed in WT animals, and the majority of the GBS were found trapped within the MMMs, with minor spreading into red pulp macrophages, probably due to direct deposit of GBS via small branches of the central artery ending in the red pulp [30] (Fig. 3A, left panel; right panel is for higher magnification). Sn-deficient spleens were still able to capture GBS in the marginal zone, which may reflect a contribution of MZMs or other pattern recognition receptors expressed on the MMMs. Notably, there were significant quantities of GBS identified around the red pulp area; most of them colocalized with the F4/80 ${ }^{+}$macrophages (Fig. 3a, middle panel), while no GBS were observed in the white pulp B cell area of either the WT or Sn-deficient spleens (Fig. 3b). An extended image set with separated channel color is available in Supplementary Fig. 2. In general, more GBS were identified in spleens of Sn- 
A $\mathrm{GBS} / \mathrm{Sn} / \mathrm{F} 4 / 80$

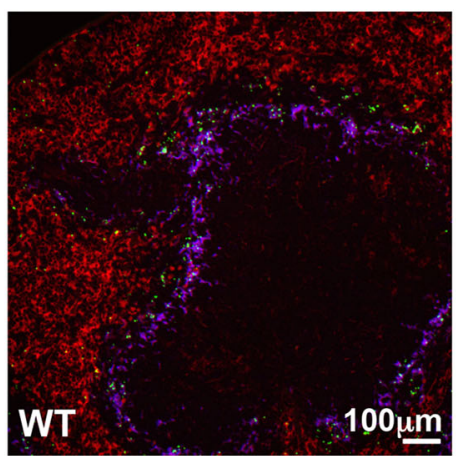

GBS/Sn/F4/80

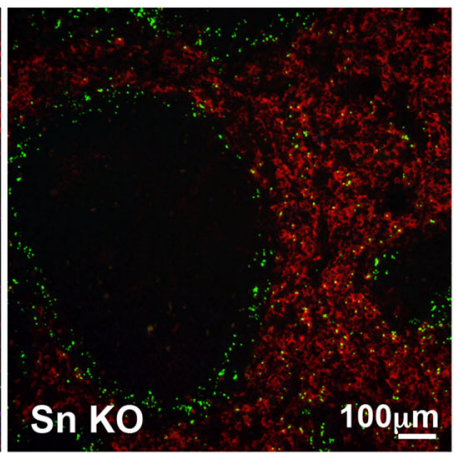

GBS/Sn/F4/80

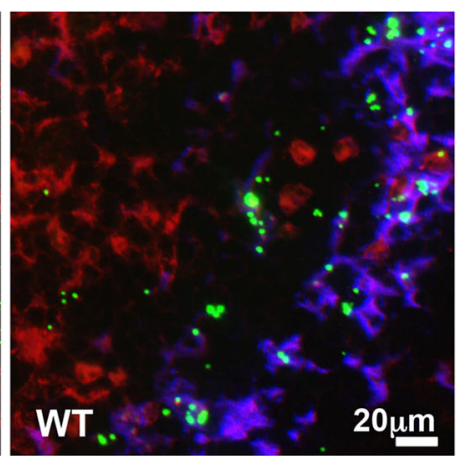

B

GBS/Sn/B220

GBS/Sn/B220
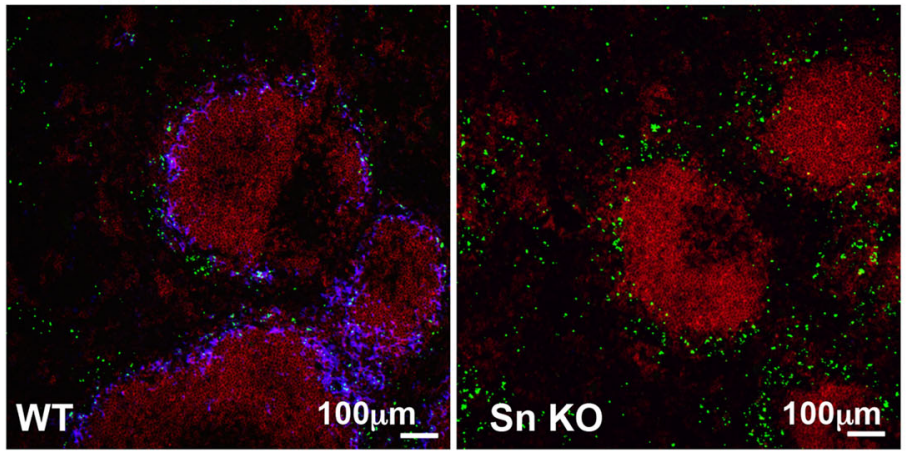

C

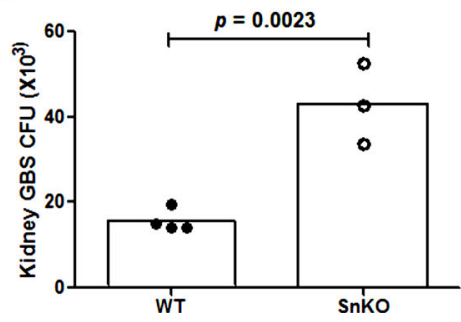

D

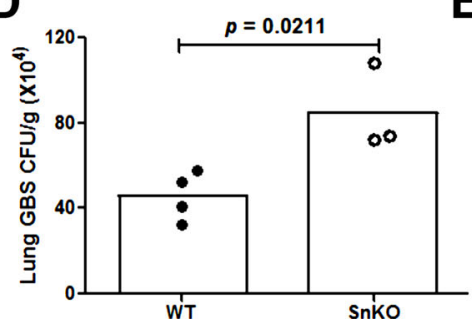

E

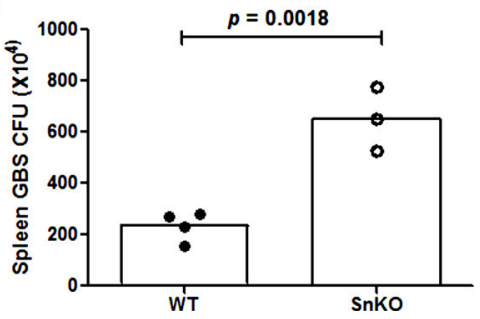

Fig. 3 Sn facilitates marginal metallophilic macrophage trapping of GBS to limit pathogen dissemination. WT and Sn-deficient mice were intravenously injected with 5-(and-6)-carboxyfluorescein labeled GBS, and kidney, lung, and spleen were collected $1 \mathrm{~h}$ post-infection. Sections were stained with mAb anti-mouse Sn, F4/80, and B220. Representative images of spleen sections are shown in $\mathbf{a}$ and $\mathbf{b}$. Scale bar is $100 \mu \mathrm{m}$ for all panels except $20 \mu \mathrm{m}$ for the right panel of a. Comparison of bacterial counts (expressed in CFU) recovered from the kidney (c), lung (d), and spleen (e) of WT mice and Sn-deficient mice $1 \mathrm{~h}$ post-infection. Difference between two groups was calculated by unpaired $t$ test. Representative data ( $n=3-4$ mice each group) were shown from two independent experiments deficient mice compared to WT animals (Fig. 3a, b), as confirmed by quantitation of GBS colony-forming unit (CFU) in the spleen (Fig. 3e). Consistent with the greater patrolling and engagement of GBS by $\mathrm{Sn}^{+}$MMMs in the WT animals, reduced GBS CFUs were recovered from the WT kidney, lung, and spleen (Fig. 3c-e). Our findings indicate that efficient capture of GBS by Sn expressed on MMMs can facilitate GBS clearance locally in the spleen and reduce the magnitude of GBS dissemination to other organs.

$\mathrm{Sn}$ is important for survival and production of anti-GBS antibodies in neutropenic mice

To date, there have been no in vivo studies addressing a role for $\mathrm{Sn}$ in controlling active infection. Since expression of $\mathrm{Sn}$ is required for macrophages to recognize and clear GBS in vitro and in vivo, we examined whether Sn could impact mouse survival upon GBS challenge. In normal mice, GBS was efficiently removed from the circulation (at least five-log reduction) within $1 \mathrm{~h}$ (Supplementary Fig. 3) after intravenous challenge. Thus, a GBS intraperitoneal challenge model was adapted to provoke systemic infection, as the bacterium can gain access to the blood circulation via this route. WT and Sndeficient mice were infected intraperitoneally with GBS, and blood CFU was enumerated $4 \mathrm{~h}$ later. As shown in Fig. 4a, greater numbers of GBS were recovered from blood of Sndeficient mice, indicating impaired bacterial clearance. However, when mouse survival was monitored through 7 days post-infection, no differences emerged between the two groups (Fig. 4b). We hypothesized more abundant and rapidly 
A
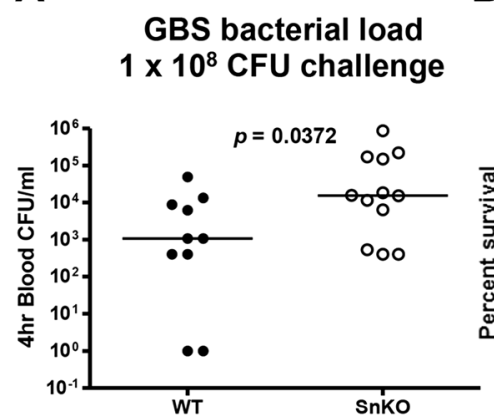

B
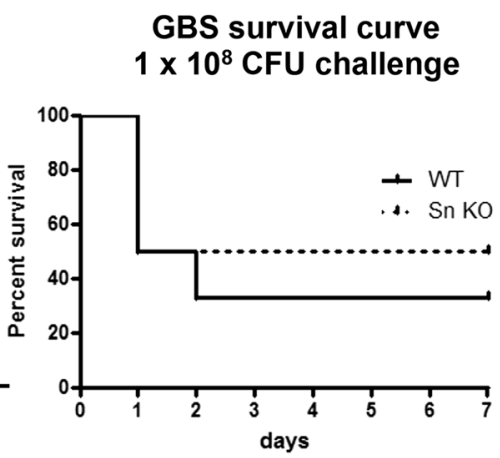

C

\section{GBS survival curve $2 \times 10^{7} \mathrm{CFU}+$ neutropenia}

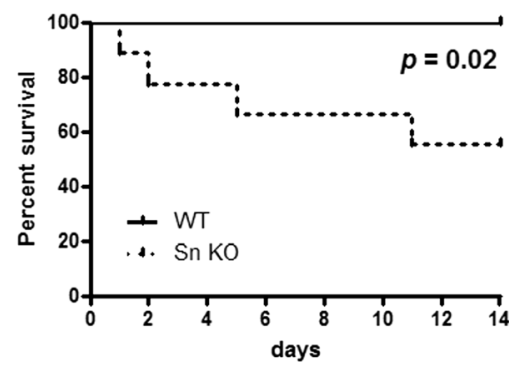

Fig. $4 \mathrm{Sn}$ is important for mouse survival during neutropenia. WT and Sn-deficient mice were intraperitoneally infected with GBS; blood was collected $4 \mathrm{~h}$ post-infection for CFU enumeration (a); and survival was monitored for 7 days (b). WT and Sn-deficient mice were intraperitoneally challenged with GBS after neutrophil depletion, and the survival was

mobilized host neutrophil response may obscure the importance of Sn in this model, since high levels of Sn expression are restricted to MMMs and SCS macrophages in the spleen and lymph nodes, respectively. To study the independent contribution of $\mathrm{Sn}$ in control of bacterial infection in the absence of neutrophils, neutrophils were depleted from mice $24 \mathrm{~h}$ prior to GBS infection. None of the WT mice died during the 14-day period, but $40 \%$ of the Sn-deficient mice died, indicating that absence of $\mathrm{Sn}$ rendered mutant mice significantly more susceptible to GBS infection under the neutropenic condition (Fig. 4c).

Sn-deficient mice were previously reported to have an intrinsic reduction in serum IgM amount [19]; therefore, we asked whether serum Ig changes could be identified following GBS challenge. As shown in Fig. 5a, serum IgM and IgG amounts were reduced in the Sn-deficient mice. Since $\mathrm{Sn}$ is critical for the phagocytosis of GBS, and $\mathrm{SCS} \mathrm{Sn}^{+}$macrophages can transfer antigens to initiate adaptive immune responses, we then further examined whether anti-GBS humoral immune responses were altered in Sn-deficient mice. We recorded for 14 days with $n=10$ for WT and $n=9$ for Sn-deficient mice (c). Long-rank test was used for statistical analysis for (b) and (c). Representative data were shown from two independent experiments

found a significant reduction of the anti-GBS IgM titer in the Sn-deficient mice (Fig. 5b), while similar anti-GBS IgG titers were observed in the two groups (Fig. 5c). Reduced antiGBS IgMs may further increase the susceptibility of Sndeficient mice to GBS infection in addition to impairment of immediate innate immune responses, such the macrophage phagocytosis and bactericidal activity.

\section{Discussion}

Efficient pathogen elimination is crucial for the host and will determine the extent and pathological consequences of infection. To combat blood-borne infectious microbes, the spleen is poised to quickly sample and trap circulating pathogens in strategically localized MZMs and MMMs [31]. These rapid innate immune mechanisms help reduce pathogen load until an adaptive immune response, more specific but temporally delayed, is mounted. Here, we provide the in vivo demonstration of Sn function in defense against an invasive sialylated

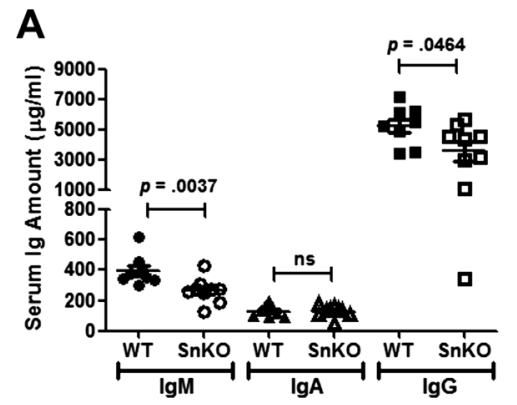

Fig. $5 \mathrm{Sn}$ is important for production of anti-GBS antibodies during neutropenia. a Decreased total IgM and IgG serum levels after GBS challenge in Sn-deficient mice. Total serum immunoglobulins from GBS-infected mice were measured by isotype specific ELISA ( $n=8$ for WT and $n=9$ for Sn-deficient mice). Mann-Whitney test was used for statistical analysis. Anti-GBS IgM (b) and anti-GBS IgG (c) titers were
B

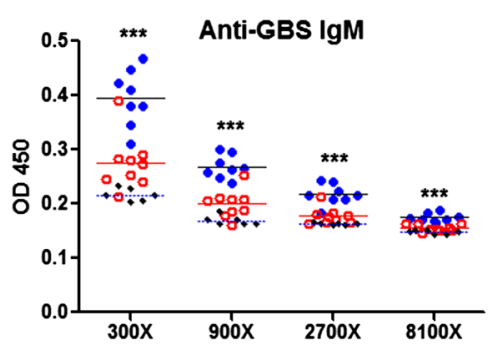

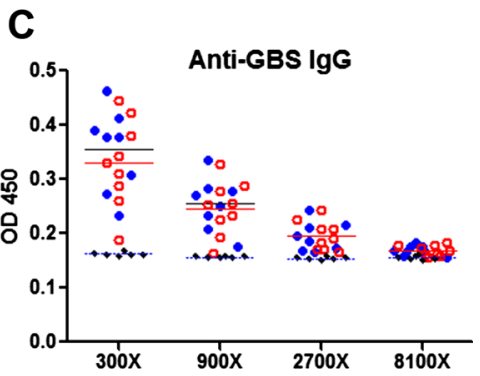

measured from GBS-infected WT (filled blue circle, $n=8$ ), GBS-infected Sn-deficient (open red circle, $n=9$ ), and uninfected WT mice (filed black circle, $n=6$ ). Difference among groups at each dilution was tested by oneway ANOVA with Tukey's multiple comparison test. $* * * P<0.001$. Representative data were shown from two independent experiments 
bacterial pathogen. This specialized macrophage Siglec not only provides innate immune functions such as phagocytosis and bactericidal activities but also supports the development of proper humoral responses against the microbe.

Most Siglecs have one or more immunoreceptor tyrosinebased inhibitory motifs (ITIMs) in the cytosolic tail and function as inhibitory receptors. Sialylated pathogens can even exploit ITIM-containing Siglecs to dampen innate immune responses therefore to enhance their survival $[18,32,33]$, in addition to reduce complement deposition and activation on the bacterial surface to impair opsonophagocytosis [20,21]. In contrast, Sn, upregulated upon infection, recognizes the very same Sia epitope and helps preserve a critical innate immune function of these phagocytic cells. As a consequence, Sn has the right properties to mediate critical initial contacts with sialylated pathogens to function directly as a phagocytic receptor or to coordinate with other pattern recognition receptors to mediate efficient phagocytosis. Our data supports this idea showing that applying anti-Sn-neutralizing mAb nearly eliminated the phagocytic ability and significantly reduced the bactericidal activity of macrophages, even as they are expected to express numerous other pattern recognition and scavenger receptors (Fig. 2b).

The humoral response provides protective circulating antibodies to neutralize or coat pathogens and facilitate subsequent opsonophagocytosis. The ability of B lymphocytes to interact with an antigen involves antigen-trapping, depositing, and transport systems localized to the major antigen entrance site. Splenic MZ macrophages can facilitate antibody production by transferring antigen captured from the circulation to MZ B cells [4, 34]. In addition, migration of splenic follicular $\mathrm{B}$ cells to the outer follicle is induced upon binding of oxysterol produced by MMMs [35-37]. SCS $\mathrm{Sn}^{+}$macrophages in the LNs transport antigens to invariant natural killer $\mathrm{T}$ cells, dendritic cells, and $\mathrm{B}$ cells and thus promote the immune responses against invaders [38-40]. Taking all the factors together, we speculate that MMMs are not only required for early antigen trapping and clearance but may also represent a crucial compartment for antigen transferring to $\mathrm{B}$ cells and follicular dendritic cells. Although Sn-deficient mice had MMMs located in the right location, they exhibited a small decrease of $\mathrm{B} 220^{+}$cells in the spleen and reduction of serum IgM levels [19]. In response to GBS challenge, Sndeficient animals showed reduced total serum IgM levels and specific anti-GBS IgM antibodies. Although the production of anti-GBS IgG antibodies was not altered in those Sn-deficient animals, they had reduced total serum IgG levels after GBS challenge. Our findings suggest that expression of Sn on MMMs is required for them to elicit specific humoral responses to counteract invading pathogens.

Whether through specific de novo biosynthesis, scavenging, or precursor scavenging, many pathogens including GBS, C. jejuni, H. influenzae, Neisseria gonorrhoeae, Neisseria meningitides, Escherichia coli K1, and Pasteurella multocida can display Sias on their cell surface as a method of molecular mimicry, counteracting complement activation and/or engaging inhibitory ITIM-bearing Siglecs on leukocytes. The specialized macrophage $\mathrm{Sn}$ receptor has a conserved binding specificity that happens to mirror the type and linkages of Sias expressed by the pathogens mentioned above. Understanding how Sn promotes Sia-dependent phagocytosis and stimulates antibody responses during such infections highlights an additional complexity in the evolutionary arms race between pathogen and host immune defense, wherein microbial glycan expression and its recognition strongly influence outcome.

Acknowledgments This work was supported by the NIH/NHLBI Programs of Excellence in Glycosciences grant P01HL107150 to A.V. and V.N. and by a Wellcome Trust Senior Fellowship WT081882 to P.R.C. We thank technical support from the UCSD Histology Core (Nissi Varki, Director) and Patrick Secrest for mouse husbandry.

Conflict of interest The authors have no financial conflict of interest with this work.

\section{References}

1. Eichner ER (1979) Splenic function: normal, too much and too little. Am J Med 66:311-320

2. Davidson RN, Wall RA (2001) Prevention and management of infections in patients without a spleen. Clin Microbiol Infect 7:657660

3. Ram S, Lewis LA, Rice PA (2010) Infections of people with complement deficiencies and patients who have undergone splenectomy. Clin Microbiol Rev 23:740-780

4. Mebius RE, Kraal G (2005) Structure and function of the spleen. Nat Rev Immunol 5:606-616

5. den Haan JM, Kraal G (2012) Innate immune functions of macrophage subpopulations in the spleen. J Innate Immun 4:437-445

6. Aichele P, Zinke J, Grode L, Schwendener RA, Kaufmann SH, Seiler P (2003) Macrophages of the splenic marginal zone are essential for trapping of blood-borne particulate antigen but dispensable for induction of specific T cell responses. J Immunol 171:1148-1155

7. Seiler P, Aichele P, Odermatt B, Hengartner H, Zinkernagel RM, Schwendener RA (1997) Crucial role of marginal zone macrophages and marginal zone metallophils in the clearance of lymphocytic choriomeningitis virus infection. Eur J Immunol 27:2626-2633

8. Rempel H, Calosing C, Sun B, Pulliam L (2008) Sialoadhesin expressed on IFN-induced monocytes binds HIV-1 and enhances infectivity. PLoS ONE 3:e1967

9. Vanderheijden N, Delputte PL, Favoreel HW, Vandekerckhove J, Van Damme J, van Woensel PA, Nauwynck HJ (2003) Involvement of sialoadhesin in entry of porcine reproductive and respiratory syndrome virus into porcine alveolar macrophages. J Virol 77:82078215

10. Zou Z, Chastain A, Moir S, Ford J, Trandem K, Martinelli E, Cicala C, Crocker P, Arthos J, Sun PD (2011) Siglecs facilitate HIV-1 infection of macrophages through adhesion with viral sialic acids. PLoS ONE 6:e24559

11. Jones C, Virji M, Crocker PR (2003) Recognition of sialylated meningococcal lipopolysaccharide by Siglecs expressed on myeloid 
cells leads to enhanced bacterial uptake. Mol Microbiol 49:12131225

12. Heikema AP, Bergman MP, Richards H, Crocker PR, Gilbert M, Samsom JN, van Wamel WJ, Endtz HP, van Belkum A (2010) Characterization of the specific interaction between sialoadhesin and sialylated Campylobacter jejuni lipooligosaccharides. Infect Immun 78:3237-3246

13. Monteiro VG, Lobato CS, Silva AR, Medina DV, de Oliveira MA, Seabra SH, de Souza W, DaMatta RA (2005) Increased association of Trypanosoma cruzi with sialoadhesin positive mouse macrophages. Parasitol Res 97:380-385

14. Klaas M, Oetke C, Lewis LE, Erwig LP, Heikema AP, Easton A, Willison HJ, Crocker PR (2012) Sialoadhesin promotes rapid proinflammatory and type I IFN responses to a sialylated pathogen, Campylobacter jejuni. J Immunol 189:2414-2422

15. Lewis AL, Nizet V, Varki A (2004) Discovery and characterization of sialic acid $O$-acetylation in group B Streptococcus. Proc Natl Acad Sci U S A 101:11123-11128

16. Weiman S, Dahesh S, Carlin AF, Varki A, Nizet V, Lewis AL (2009) Genetic and biochemical modulation of sialic acid $O$-acetylation on group B Streptococcus: phenotypic and functional impact. Glycobiology 19:1204-1213

17. Crocker PR, Gordon S (1989) Mouse macrophage hemagglutinin (sheep erythrocyte receptor) with specificity for sialylated glycoconjugates characterized by a monoclonal antibody. J Exp Med 169:1333-1346

18. Carlin AF, Uchiyama S, Chang YC, Lewis AL, Nizet V, Varki A (2009) Molecular mimicry of host sialylated glycans allows a bacterial pathogen to engage neutrophil Siglec-9 and dampen the innate immune response. Blood 113:3333-3336

19. Oetke C, Vinson MC, Jones C, Crocker PR (2006) Sialoadhesindeficient mice exhibit subtle changes in $\mathrm{B}$ - and T-cell populations and reduced immunoglobulin M levels. Mol Cell Biol 26:1549-1557

20. Rubens CE, Wessels MR, Heggen LM, Kasper DL (1987) Transposon mutagenesis of type III group B Streptococcus: correlation of capsule expression with virulence. Proc Natl Acad Sci U S A 84:7208-7212

21. Wessels MR, Rubens CE, Benedi VJ, Kasper DL (1989) Definition of a bacterial virulence factor: sialylation of the group B streptococcal capsule. Proc Natl Acad Sci U S A 86:8983-8987

22. Marques MB, Kasper DL, Pangburn MK, Wessels MR (1992) Prevention of $\mathrm{C} 3$ deposition by capsular polysaccharide is a virulence mechanism of type III group B streptococci. Infect Immun 60:39863993

23. Vimr E, Lichtensteiger C (2002) To sialylate, or not to sialylate: that is the question. Trends Microbiol 10:254-257

24. York MR, Nagai T, Mangini AJ, Lemaire R, van Seventer JM, Lafyatis R (2007) A macrophage marker, Siglec-1, is increased on circulating monocytes in patients with systemic sclerosis and induced by type I interferons and Toll-like receptor agonists. Arthritis Rheum 56:1010-1020

25. Bao G, Han Z, Yan Z, Wang Q, Zhou Y, Yao D, Gu M, Chen B, Chen S, Deng A et al (2010) Increased Siglec-1 expression in monocytes of patients with primary biliary cirrhosis. Immunol Invest 39:645-660
26. Biesen R, Demir C, Barkhudarova F, Grun JR, Steinbrich-Zollner M, Backhaus M, Haupl T, Rudwaleit M, Riemekasten G, Radbruch A et al (2008) Sialic acid-binding Ig-like lectin 1 expression in inflammatory and resident monocytes is a potential biomarker for monitoring disease activity and success of therapy in systemic lupus erythematosus. Arthritis Rheum 58:1136-1145

27. Cornelissen M, van der Kuyl AC, van den Burg R, Zorgdrager F, van Noesel CJ, Goudsmit J (2003) Gene expression profile of AIDSrelated Kaposi's sarcoma. BMC Cancer 3:7

28. van der Kuyl AC, van den Burg R, Zorgdrager F, Groot F, Berkhout B, Cornelissen M (2007) Sialoadhesin (CD169) expression in CD14+ cells is upregulated early after HIV-1 infection and increases during disease progression. PLoS ONE 2:e257

29. Xiong YS, Zhou YH, Rong GH, Wu WL, Liang Y, Yang ZX, Geng HL, Zhong RQ (2009) Siglec-1 on monocytes is a potential risk marker for monitoring disease severity in coronary artery disease. Clin Biochem 42:1057-1063

30. Kraal G (1992) Cells in the marginal zone of the spleen. Int Rev Cytol 132:31-74

31. Junt T, Moseman EA, Iannacone M, Massberg S, Lang PA, Boes M, Fink K, Henrickson SE, Shayakhmetov DM, Di Paolo NC et al (2007) Subcapsular sinus macrophages in lymph nodes clear lymph-borne viruses and present them to antiviral B cells. Nature 450:110-114

32. Cao H, Crocker PR (2011) Evolution of CD33-related siglecs: regulating host immune functions and escaping pathogen exploitation? Immunology 132:18-26

33. Khatua B, Bhattacharya K, Mandal C (2012) Sialoglycoproteins adsorbed by Pseudomonas aeruginosa facilitate their survival by impeding neutrophil extracellular trap through Siglec-9. J Leukoc Biol 91:641-655

34. Kraal G, Mebius R (2006) New insights into the cell biology of the marginal zone of the spleen. Int Rev Cytol 250:175-215

35. Pereira JP, Kelly LM, Cyster JG (2010) Finding the right niche: Bcell migration in the early phases of T-dependent antibody responses. Int Immunol 22:413-419

36. Hannedouche S, Zhang J, Yi T, Shen W, Nguyen D, Pereira JP, Guerini D, Baumgarten BU, Roggo S, Wen B et al (2011) Oxysterols direct immune cell migration via EBI2. Nature 475: 524-527

37. Liu C, Yang XV, Wu J, Kuei C, Mani NS, Zhang L, Yu J, Sutton SW, Qin N, Banie H et al (2011) Oxysterols direct B-cell migration through EBI2. Nature 475:519-523

38. Barral P, Polzella P, Bruckbauer A, van Rooijen N, Besra GS, Cerundolo V, Batista FD (2010) CD169(+) macrophages present lipid antigens to mediate early activation of iNKT cells in lymph nodes. Nat Immunol 11:303-312

39. Asano K, Nabeyama A, Miyake Y, Qiu CH, Kurita A, Tomura M, Kanagawa O, Fujii S, Tanaka M (2011) CD169-positive macrophages dominate antitumor immunity by crosspresenting dead cellassociated antigens. Immunity 34:85-95

40. Martinez-Pomares L, Gordon S (2012) CD169+ macrophages at the crossroads of antigen presentation. Trends Immunol 33: $66-70$ 